\title{
Notes on the bryophyte flora and vegetation of the central and south-western Balkans
}

\author{
Marta Puglisi (*), Patrizia Campisi (**), Dmitar Lakušić (***), \\ Boštjan Surina (****), Romeo Di Pietro (*****), Maria Privitera (*)
}

\begin{abstract}
Puglisi, M., Campisi, P., Lakušić, D., Surina, B., Di Pietro, R., Privitera, M. Notes on the bryophyte flora and vegetation of the central and south-western Balkans. Lazaroa 34: 107-116 (2013).

A study on the bryophyte flora and vegetation was carried out in the mountains at the boundary between Albania, Macedonia and Montenegro. The study area included Maja and Jezercës massif (Prokletije mts., SE Dinaric Alps) and Mt Korab (Šar-Pindos Range) in Macedonia. Several records for the bryological flora of Macedonia and Albania are reported. In particular Scapania cuspiduligera and Distichium inclinatum are new records for the Albanian flora. In addition some bryophytic and bryo-chormophytic associations belonging to the phytosociological classes Ctenidietea mollusci and Montio fontanae-Cardaminetea amarae are reported too.
\end{abstract}

Keywords: Bryophytes, Dinaric Alps, Šar-Pindos range, Macedonia, Montenegro.

Resumen: Puglisi, M., Campisi, P., Lakušić, D., Surina, B., Di Pietro, R., Privitera, M. Notas sobre la brioflora y su vegetación del centro y suroeste de los Balcanes. Lazaroa 34: 107-116 (2013).

Presentamos un estudio sobre la flora y la vegetación briofítica de los Balcanes, en concreto de aquellos macizos montañosos que se encuentran entre Albania, Macedonia y Montenegro. La zona de estudio incluye las montañas de Maja y el macizo de Jezercës (Montes Prokletije, sureste de los Alpes Dináricos), así como los montes Korab (Cordillera Šar-Pindos) en Macedonia. El material briofítico recolectado ha resultado de gran interés para la brioflora de Macedonia y Albania. En concreto Scapania cuspiduligera y Distichium inclinatum se consideran nuevas citas para Albania. Además algunas asociaciones briofíticas y briocormofíticas de Ctenidietea mollusci y Montio fontanae-Cardaminetea amarae se han considerado también en este trabajo.

Palabras clave: Briófitos, Alpes Dináricos, Cordillera Šar-Pindos, Macedonia, Montenegro.

\section{INTRODUCTION}

From a bioclimatic and biogeographic viewpoint, the Balkan Peninsula is considered one of the most interesting areas in the whole southeastern Europe. In particular the study area, composed of the Prokletije range and part of the Šar-Pindos range in Macedonia comprises some of the highest peaks of the whole Balkans range preserving a high number of circumboreal/Arctic species that spread southwards during the Quaternary glaciations. The first studies on the Flora of the southern Dinaric Alps were carried out by GRISEBACH $(1843,1844)$ and continued by other authors in the first half of the twentieth century (e.g. Degen \& Dörfler, 1897; HayeK, 1917; BORNMÜLLER, 1933; KoŠANIN, 1939). In the last two decades many studies focused on the flora of the alpine belt (e.g. VANGJELI \& al., 2000; STEVANOVIĆ \& al., 2003, 2009; LAKUŠIĆ \& al., 2004; BARINA \& PIFKO, 2008; RAKAJ, 2009) where several new species were described.

While the flowering plants have been studied from the $19^{\text {th }}$ century onwards, the knowledge on

\footnotetext{
* Department of Biological. Geological and Environmental Sciences. University of Catania, Italy. E-mail: mpuglisi@unict.it

** Department of Biological. Chemical and Pharmaceutical Sciences and Technologies. University of Palermo, Italy.

*** Botanical Institute and Garden. Faculty of Biology. University of Belgrade. 11000. Belgrade, Serbia.

****University of Primorska. Faculty of Mathematics. Natural Sciences and Information Technologies. SI-6000 Koper, Slovenia ***** PDTA Department. Sapienza University of Rome, Italy.
} 
the bryophyte flora, particularly from the SE Dinaric Alps, is still rather incomplete and the available data are mostly scattered. Amongst the Balkan countries, Macedonia and Albania are the most poorly known due to the scarcity of investigations and the very low number of local bryologists. The first bryofloristic reports date back to the late 1800's for Albania (BECK \& SZYSZYLOWICZ, 1888) and to the beginning of 1900's for Macedonia (Herzog, 1919). More recent information can be drawn from Düll (1983, 1984, 1985, 1992), TSAKIRI \& al. (1998), DÜll \& al. (1999), SÖDERSTRÖM \& al. (2002), PAPP \& al. (2010), etc. The increase of the activity in bryophyte investigation which took place during the last decade has resulted in an increase of the bryological records at regional scale. The bryophyte data have been then assembled in a preliminary checklist published by COLACINO \& SABOVlJević (2006) for Albania, and in the different checklists recently issued on the bryophytes of South-Eastern Europe or the Mediterranean region (SABOVluević \& al. 2001, 2008; Ros \& al. $2007,2013)$. As far as the number of hornworts, liverworts and moss taxa is concerned, Albania counts for 3, 89 and 258 respectively, and Macedonia for 1, 71 (all referable to old reports) and 403 respectively. In the present paper, the preliminary bryological results of a wider botanical investigation aimed to improve the knowledge of the Balkan high-altitude flora and vegetation of vascular plants and bryophytes are presented.

The field work was carried out in July 2011. The nomenclature of the species follows Ros \& al. (2013) for the mosses and Ros \& al. (2007) for the liverworts. The chorotype is reported according to HiLL \& al. (2007). Names of the vascular plants follow Euro+Med Plant base (2006).

The study on bryophyte vegetation followed the plant sociological method of BRAUN-BLANQUET (1964) applied to the bryophytes (PUGLISI, 2008). The bryological synsystematic and syntaxonomy follows MARSTALLER (2006) according to the ICPN of WEBER \& al. (2000).

The exsiccata are kept in the Herbarium of the Department of Biological, Geological and Environmental Sciences of the University of Catania (CAT).

\section{MATERIALS AND METHODS}

\section{STUDY AREA}

Collecting sites refer to the Prokletije mountains (a triple boundary between Albania, Kosovo and Montenegro) in south-easternmost Dinaric Alps, and the second ones are located in Mt Korab (Macedonia), part of the Šar-Pindos mountain range (Figure 1). The Prokletije mountains, which are also known as Albanian Alps (Bjeshket e Namuna) extend from northern Albania to Kosovo and eastern Montenegro. Maja e Jezercës Peak (2,694 m), in Albania, represents the highest summit of the whole Dinaric Alps. Korab Range (the highest peak is Golem Korab Mt., 2,764 m; Maja e Korabit) is a very rugged mountain massif located at the border between the Republics of Macedonia and Albania. From a lithological viewpoint the Prokletije Mountains are mainly composed of Mesozoic limestones and dolomites of the Jurassic and Cretaceous Ages while Korab Mt. exhibits a more intricate situation that provides other bedrock types in addition to the dominant Palaeozoic and Triassic period limestone (VRANAJ, 1990). The climate in the high altitude zones is cold and snow is currently found even in summer. The Prokletije Mts are one of the wettest areas of Europe where even the driest valleys exhibit rainfalls of 2,000 millimeters per year (OBULJEN, 1962; FILIPOVSKI \& al., 1996).

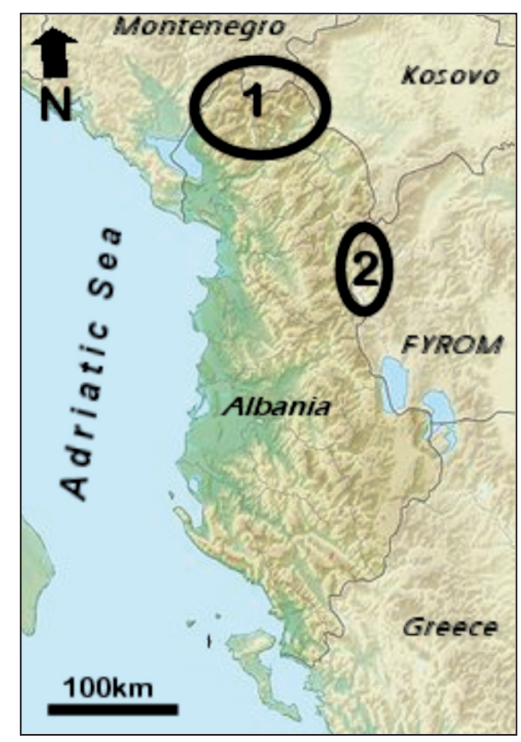

Figure 1.-Study area. 1: Prokletije mountains; 2: Mount Korab. 


\section{Collecting SiTES}

- Dinaric Alps, Prokletije mountain Range, Maja e Jezercës Mt. (Albania); 2300 m. chasmophytic vegetation on limestone rocks, partially shaded sites, $42^{\circ} 26^{\prime} 45.60^{\prime \prime} \mathrm{N}, 19^{\circ} 48^{\prime} 46.80^{\prime \prime} \mathrm{E}$ (Leg. R. Di Pietro, 27.07.2011).

- Dinaric Alps, Prokletije Range, Maja e Jezercës Mt. (Albania); $2450 \mathrm{~m}$. chasmophytic vegetation on limestone rocks, $42^{\circ} 26^{\prime} 4.58^{\prime \prime} \mathrm{N} 19^{\circ} 49^{\prime} 6.77 \mathrm{E}$ (Leg. R. Di Pietro, 27.07.2011).

- Dinaric Alps, Prokletije range, Buni Jezercës (Albania); $1850 \mathrm{~m}$. calcareous spring, $42^{\circ} 27^{\prime 2} 26.64 " \mathrm{~N}$, 1948'29.88"E (Leg. R. Di Pietro, 27.07.2011).

- Šar-Pindos range, Mt. Golem Korab (Republic of Macedonia): $2600 \mathrm{~m}$, shaded calcareous spring, 414' $9.62^{\prime \prime} \mathrm{N} 20^{\circ} 32^{\prime} 56.47^{\prime \prime} \mathrm{E}$ (Leg. R. Di Pietro, 22.07.2011).

\section{RESULTS AND DISCUSSION}

\section{BRYOPHYTE FLORA}

The list with the bryophytes collected in the study areas is reported below. Families and species within each family are listed in alphabetical order; for each species the chorotype and the sites of collection are provided. Explanation of symbols:

** new record for Albania.

\# second record for Albania.

* confirmation of records in Albania or Macedonia previous to 1962 .

\section{Liverworts}

\section{Scapaniaceae}

** Scapania cuspiduligera (Nees) Müll. Frib.

Albania: Dinaric Alps, Prokletije range, Buni Jezercës, $1850 \mathrm{~m}$, calcareous spring, $42^{\circ} 27^{\prime} 26.64 " \mathrm{~N}$, $19^{\circ} 48^{\prime} 29.88^{\prime \prime}$ E, R. Di Pietro, 27. 07. 2011, CAT 03.11/ALB.

Circumpolar-Boreo-Arctic-Montane.

Mosses

\section{Amblystegiaceae}

* Cratoneuron filicinum (Hedw.) Spruce

Albania: Dinaric Alps, Prokletije range, Buni Jezercës, $1850 \mathrm{~m}$, calcareous spring, $42^{\circ} 27^{\prime} 26.64 " \mathrm{~N}$, 1948'29.88"E, R. Di Pietro, 27.07.2011, CAT 05.11/ALB. MACEDONIA: Šar-Pindos range, Mt. Golem Korab, 2600 m, shaded calcareous spring, $41^{\circ} 47^{\prime} 9.62^{\prime \prime} \mathrm{N} 20^{\circ} 32^{\prime} 56.47^{\prime \prime} \mathrm{E}, \mathrm{R}$. Di Pietro, 22.07.2011, CAT 30.11/MAC.

Circumpolar-wide-Temperate.

\section{* Palustriella decipiens (De Not.) Ochyra}

Albania: Dinaric Alps, Prokletije range, Buni Jezercës, $1850 \mathrm{~m}$, calcareous spring, $42^{\circ} 27^{\prime} 26.64^{\prime \prime} \mathrm{N}$, 1948'29.88"E, R. Di Pietro, 27.07.2011, CAT 01.11/ALB.

European Boreo-Arctic-Montane.

\section{* Palustriella commutata (Hedw.) Ochyra}

Albania: Dinaric Alps, Prokletije range, Buni Jezercës, $1850 \mathrm{~m}$, calcareous spring, $42^{\circ} 27^{\prime} 26.64 " \mathrm{~N}$, 1948'29.88"E, R. Di Pietro, 27.07.2011, CAT 04.11/ALB. MaCEDONIA: Šar-Pindos range, Mt. Golem Korab, 2600 m, shaded calcareous spring, $41^{\circ} 47^{\prime} 9.62 " \mathrm{~N} 20^{\circ} 32^{\prime} 56.47^{\prime \prime} \mathrm{E}, \mathrm{R}$. Di Pietro, 22. 7. 2011, CAT 37.11/MAC.

Circumpolar-Boreo-Temperate.

\section{Bartramiaceae}

Philonototis caespitosa Jur.

MACEDONIA: Šar-Pindos range, Mt. Golem Korab, $2600 \mathrm{~m}$, shaded calcareous spring, $41^{\circ} 47^{\prime} 9.62^{\prime \prime} \mathrm{N}$ 20³2'56.47"E, R. Di Pietro, 22.07.2011, CAT 32.11/MAC.

Circumpolar-Boreo-Temperate.

\section{Bryaceae}

\# Bryum dichotomum Hedw.

Albania: Dinaric Alps, Prokletije mountain Range, Maja e Jezercës Mt., 2300 m. chasmophytic vegetation on limestone rocks, partially shaded sites, 42²6'45.60"N, 1948'46.80"E, R. Di Pie- 
tro, 27.07.2011), CAT 11.11/ALB. Dinaric Alps, Prokletije Range, Maja e Jezercës Mt., 2450 m., chasmophytic vegetation on limestone rocks, $42^{\circ} 26^{\prime} 4.58^{\prime \prime N} 19^{\circ} 49^{\prime} 6.77 " \mathrm{E}, \quad \mathrm{R}$. Di Pietro, 27.07.2011, CAT 19.11/ALB. Dinaric Alps, Prokletije range, Buni Jezercës, $1850 \mathrm{~m}$, calcareous

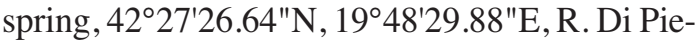
tro, 27. 7. 2011, CAT 02.11/ALB.

European-wide-Temperate.

Bryum schleicheri DC.

MACEDONIA: Šar-Pindos range, Mt. Golem Korab, $2600 \mathrm{~m}$, shaded calcareous spring, $41^{\circ} 47^{\prime} 9.62^{\prime \prime} \mathrm{N}$ 20³2'56.47"E, R. Di Pietro, 22.07.2011, CAT 38.11/MAC.

Circumpolar-Boreal-Montane.

*Ptychostomum pseudotriquetrum (Hedw.) J. R. Spence \& H. P. Ramsay var. pseudotriquetrum

Albania: Dinaric Alps, Prokletije range, Buni Jezercës, $1850 \mathrm{~m}$, calcareous spring, $42^{\circ} 27^{\prime} 26.64 " \mathrm{~N}$, 1948'29.88"E, R. Di Pietro, 27.07.2011, CAT 06.11/ALB. MACEDONIA: Šar-Pindos range, Mt. Golem Korab, 2600 m, shaded calcareous spring, $41^{\circ} 47^{\prime} 9.62^{\prime \prime} \mathrm{N} 20^{\circ} 32^{\prime} 56.47^{\prime \prime E}$, R. Di Pietro, 22.07.2011, CAT 31.11/MAC.

Circumpolar-wide-Boreal.

\section{Ditrichaceae}

*Distichium capillaceum (Hedw.) Bruch \& Schimp.

Albania: Dinaric Alps, Prokletije mountain Range, Maja e Jezercës Mt., 2300 m. chasmophytic vegetation on limestone rocks, partially shaded sites, $42^{\circ} 26^{\prime} 45.60 " \mathrm{~N}, 1^{\circ} 48^{\prime} 46.80^{\prime \prime} \mathrm{E}, \mathrm{R}$. Di Pietro, 27.07.2011), CAT 12.11/ALB. Dinaric Alps, Prokletije Range, Maja e Jezercës Mt., 2450 m. chasmophytic vegetation on limestone rocks, $42^{\circ} 26^{\prime} 4.58 " \mathrm{~N} \quad 9^{\circ} 49^{\prime} 6.77^{\prime \prime E}, \quad \mathrm{R}$. Di Pietro, 27.07.2011, CAT 18.11/ALB. MACEDONIA: ŠarPindos range, Mt. Golem Korab, 2600 m, shaded calcareous spring, $41^{\circ} 47^{\prime} 9.62^{\prime \prime} \mathrm{N} 20^{\circ} 32 ' 56.47 " \mathrm{E}$, R. Di Pietro, 22.07.2011, CAT 33.11/MAC. Circumpolar-Boreal-Temperate.
**Distichium inclinatum (Hedw.) Bruch \& Schimp.

Albania: Dinaric Alps, Prokletije range, Buni Jezercës, $1850 \mathrm{~m}$, calcareous spring, $42^{\circ} 27^{\prime} 26.64^{\prime \prime} \mathrm{N}$, 1948'29.88"E, R. Di Pietro, 27.07.2011, CAT s.n. Circumpolar-Boreo-Arctic-Montane.

\section{Encalyptaceae}

* Encalypta streptocarpa Hedw.

Albania: Dinaric Alps, Prokletije mountain Range, Maja e Jezercës Mt., 2300 m. chasmophytic vegetation on limestone rocks, partially shaded sites, $42^{\circ} 26^{\prime} 45.60^{\prime \prime} \mathrm{N}, 19^{\circ} 48^{\prime} 46.80^{\prime \prime} \mathrm{E}, \mathrm{R}$. Di Pietro, 27.07.2011, CAT 13.11/ALB. Dinaric Alps, Prokletije Range, Maja e Jezercës Mt., 2450 m. chasmophytic vegetation on limestone rocks, 42 $26^{\prime} 4.58^{\prime \prime N} 19^{\circ} 49^{\prime} 6.77^{\prime \prime E}, \quad R . \quad$ Di Pietro, 27.07.2011, CAT 17.11/ALB. Dinaric Alps, Prokletije range, Buni Jezercës, $1850 \mathrm{~m}$, calcareous spring, 42²7'26.64"N, 1948'29.88"E, R. Di Pietro, 27. 7. 2011, CAT 39.11/ALB.

Eurasian-Boreo-Temperate.

\section{Fissidentaceae}

Fissidens dubius P. Beauv.

Albania: Dinaric Alps, Prokletije Range, Maja e Jezercës Mt.,2450 m. chasmophytic vegetation on limestone rocks, $42^{\circ} 26^{\prime} 4.58^{\prime \prime} \mathrm{N} 19^{\circ} 49^{\prime} 6.77 " \mathrm{E}, \mathrm{R}$. Di Pietro, 27.07.2011, CAT 20.11/ALB. Dinaric Alps, Prokletije range, Buni Jezercës, $1850 \mathrm{~m}$, calcareous spring, $42^{\circ} 27^{\prime} 26.64 " \mathrm{~N}, 1^{\circ} 48^{\prime} 29.88^{\prime \prime} \mathrm{E}, \mathrm{R}$. Di Pietro, 27.07.2011, CAT 25.11/ALB.

European-Temperate.

\section{Leskeaceae}

Leskea polycarpa Hedw.

MACEDONIA: Šar-Pindos range, Mt. Golem Korab, $2600 \mathrm{~m}$, shaded calcareous spring, $41^{\circ} 47^{\prime} 9.62^{\prime \prime} \mathrm{N}$ $20^{\circ} 32^{\prime} 56.47 " \mathrm{E}, \mathrm{R}$. Di Pietro, 22.07.2011, CAT 35.11/MAC.

Circumpolar-Temperate. 
Pseudoleskea incurvata (Hedw.) Loeske

AlbANIA: Dinaric Alps, Prokletije mountain Range, Maja e Jezercës Mt., 2300 m., chasmophytic vegetation on limestone rocks, partially shaded sites, 42 $26^{\prime} 45.60^{\prime \prime} \mathrm{N}, 1^{\circ} 48^{\prime} 46.80^{\prime \prime} \mathrm{E}, \mathrm{R}$. Di Pietro, 27.07.2011,CAT 14.11/ALB. Dinaric Alps, Prokletije Range, Maja e Jezercës Mt., 2450 m. chasmophytic vegetation on limestone rocks, $42^{\circ} 26^{\prime} 4.58^{\prime \prime} \mathrm{N}$ 1949'6.77"E, R. Di Pietro, 27.07.2011, CAT 21.11/ALB. Dinaric Alps, Prokletije range, Buni Jezercës, $1850 \mathrm{~m}$, calcareous spring, 42²7'26.64"N, $19^{\circ} 48$ '29.88"E, R. Di Pietro, 27. 7. 2011, CAT 27.11/ALB

European Boreal-Montane.

\section{\# Pseudoleskeella nervosa (Brid.) Nyholm}

Albania: Dinaric Alps, Prokletije range, Buni Jezercës, $1850 \mathrm{~m}$, calcareous spring, $42^{\circ} 27^{\prime} 26.64 " \mathrm{~N}$, 1948'29.88"E, R. Di Pietro, 27.07.2011, CAT 08.11/ALB.

Circumpolar Boreal-Montane.

\section{Mniaceae}

* Mnium stellare Hedw.

Albania: Dinaric Alps, Prokletije mountain Range, Maja e Jezercës Mt., 2300 m. chasmophytic vegetation on limestone rocks, partially shaded

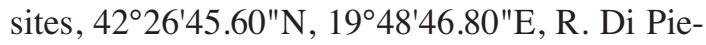
tro, 27.07.2011, CAT 15.11/ALB. Dinaric Alps, Prokletije Range, Maja e Jezercës Mt., 2450 m., chasmophytic vegetation on limestone rocks,

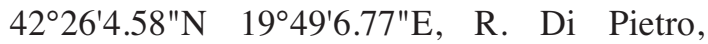
27.07.2011, CAT 22.11/ALB.

Circumpolar Boreal-Temperate.

* Mnium thomsonii Schimp.

Albania: Dinaric Alps, Prokletije Range, Maja e Jezercës Mt., 2450 m., chasmophytic vegetation on limestone rocks, $42^{\circ} 26^{\prime} 4.58^{\prime \prime} \mathrm{N} 19^{\circ} 49^{\prime} 6.77 " \mathrm{E}$, R. Di Pietro, 27.07.2011, CAT 26.11/ALB. Circumpolar Boreo-Arctic-Montane.

\section{Polytrichaceae}

* Polytrichum commune Hedw.

MACEDOnIA: Šar-Pindos range, Mt. Golem Korab, $2600 \mathrm{~m}$, shaded calcareous spring, $41^{\circ} 47^{\prime} 9.62^{\prime \prime} \mathrm{N}$ 20³2'56.47"E, R. Di Pietro, 22.07.2011, CAT 34.11/MAC.

Circumpolar wide Boreal.

\section{Polytrichum juniperinum Hedw}

MACEDOnIA: Šar-Pindos range, Mt. Golem Korab, $2600 \mathrm{~m}$, shaded calcareous spring, $41^{\circ} 47^{\prime} 9.62^{\prime \prime} \mathrm{N}$ 20³2'56.47"E, R. Di Pietro, 22.07.2011, CAT 28.11/MAC.

Circumpolar wide Boreal.

\section{Pottiaceae}

* Didymodon fallax (Hedw.) R. H. Zander

Albania: Dinaric Alps, Prokletije range, Buni Jezercës, $1850 \mathrm{~m}$, calcareous spring, $42^{\circ} 27^{\prime} 26.64 " \mathrm{~N}$, 1948'29.88"E, R. Di Pietro, 27.07.2011, CAT 09.11/ALB.

Circumpolar Southern-Temperate.

* Gymnostonum aeruginosum $\mathrm{Sm}$.

Albania: Dinaric Alps, Prokletije mountain Range, Maja e Jezercës Mt., 2300 m., chasmophytic vegetation on limestone rocks, partially shaded sites, $42^{\circ} 26^{\prime} 45.60^{\prime \prime} \mathrm{N}, 1^{\circ} 48^{\prime} 46.80^{\prime \prime} \mathrm{E}, \mathrm{R}$. Di Pietro, 27.07.2011, CAT 16.11/ALB. Dinaric Alps, Prokletije Range, Maja e Jezercës Mt., 2450 $\mathrm{m}$., chasmophytic vegetation on limestone rocks, $42^{\circ} 26^{\prime} 4.58^{\prime \prime N} 19^{\circ} 49^{\prime} 6.77^{\prime \prime E}, \quad R . \quad$ Di Pietro, 27.07.2011, CAT 23.11/ALB. MACEDONIA: ŠarPindos range, Mt. Golem Korab, 2600 m, shaded calcareous spring, $41^{\circ} 47^{\prime} 9.62^{\prime \prime} \mathrm{N} 20^{\circ} 32^{\prime} 56.47 " \mathrm{E}$, R. Di Pietro, 22.07.2011, CAT 36.11/MAC. Circumpolar Boreal-Temperate.

\section{Syntrichia norvegica F. Weber}

Albania: Dinaric Alps, Prokletije Range, Maja e Jezercës Mt., 2450 m., chasmophytic vegetation on limestone rocks, $42^{\circ} 26^{\prime} 4.58^{\prime \prime} \mathrm{N} 19^{\circ} 49^{\prime} 6.77 " \mathrm{E}$, R. Di Pietro, 27.07.2011, CAT 28.11/ALB. 
Circumpolar Arctic-Montane.

Tortella tortuosa (Hedw.) Limpr.

Albania: Dinaric Alps, Prokletije Range, Maja e Jezercës Mt., 2450 m., chasmophytic vegetation on limestone rocks, $42^{\circ} 26^{\prime} 4.58^{\prime \prime} \mathrm{N} 19^{\circ} 49^{\prime} 6.77 " \mathrm{E}$, R. Di Pietro, 27.07.2011, CAT 24.11/ALB. Dinaric Alps, Prokletije range, Buni Jezercës, $1850 \mathrm{~m}$, calcareous spring, $42^{\circ} 27^{\prime} 26.64^{\prime \prime} \mathrm{N}, 19^{\circ} 48^{\prime} 29.88^{\prime \prime} \mathrm{E}$, R. Di Pietro, 27.07.2011, CAT 10.11/ALB. MACEDONIA: Šar-Pindos range, Mt. Golem Korab, $2600 \mathrm{~m}$, shaded calcareous spring, $41^{\circ} 47^{\prime} 9.62^{\prime \prime} \mathrm{N}$ 20³2'56.47"E, R. Di Pietro, 22.07.2011, CAT 29.11/MAC.

Circumpolar Boreal-Temperate.

Twenty-three bryophytes have been collected in the study areas: 1 liverwort and 22 mosses. These species mostly belong to a typical orophytic, terri-saxicolous and selected bryoflora that has its distribution centre in the montane areas of Central Europe and are found scattered in isolated sites within the high mountains of the southern European Countries (Puglisi \& al., 2012; PUGLISI \&al., 2013a).

Amongst the surveyed species it is worth noting the discovery of the liverwort Scapania cuspiduligera, a new record for Albania; this is a
Circumpolar Boreo-Arctic montane species, very rare in the Mediterranean area where it only occurs in Spain, France and Italy, while the old records for Slovenia and Greece are still to be confirmed (Ros \& al., 2007). Another new record from Albania is the moss Distichium inclinatum, also a Circumpolar Boreo-Arctic montane species. Furthermore we confirm some old reports (Polytrichum commune for Macedonia, and Leskea polycarpa, Mnium thomsonii, Palustriella decipiens and Syntrichia norvegica for Albania), whose previous records were based on collections published before 1962 (Ros \& al., 2013).

As already evidenced in other Mediterranean montane areas (Privitera \& PUGLISI, 1997, 2002; Puglisi, 2009; Puglisi \& al. 2009, 2011), also in the southwestern part of the Dinaric Alps the alpine belt is currently playing the role of refuge for many relict boreal-montane and arctic-montane species and communities that colonized the southern Europe during the glaciations. The high altitude of the main peaks allows these mountains to play an important role in terms of biodiversity conservation and to represent important sites for the maintaining and safeguard of the bryophyte diversity, as already highlighted for other southEuropean territories (Privitera \& Puglisi, 2009; Puglisi, 2009, 2010; Puglisi \& al. 2011, 2013b).

\begin{tabular}{|c|c|c|c|c|c|}
\hline \multicolumn{6}{|c|}{$\begin{array}{c}\text { Table } 1 \\
\text { Pseudoleskeetum incurvatae Ježek \& Vondráček } 1962 \\
\text { (Ctenidion mollusci, Ctenidietalia mollusci, Ctenidietea mollusci) }\end{array}$} \\
\hline Altitude (m) & 2450 & 2450 & 2450 & 2450 & 1850 \\
\hline Size of relevé $\left(\mathrm{dm}^{2}\right)$ & 15 & 10 & 10 & 10 & 20 \\
\hline Cover $(\%)$ & 85 & 55 & 75 & 45 & 60 \\
\hline Inclination $\left({ }^{\circ}\right)$ & 5 & 10 & - & 5 & 5 \\
\hline Exposure & $\mathrm{NE}$ & $\mathrm{NE}$ & - & $\mathrm{NE}$ & $\mathrm{E}$ \\
\hline Relevé number & 1 & 2 & 3 & 4 & 5 \\
\hline \multicolumn{6}{|l|}{ Characteristics } \\
\hline Pseudoleskea incurvata & 4 & 3 & 4 & 2 & 3 \\
\hline Tortella tortuosa & 2 & . & + & 2 & 1 \\
\hline Encalypta streptocarpa & . & 1 & . & . & + \\
\hline Fissidens dubius & . & 1 & . & + & 1 \\
\hline $\begin{array}{l}\text { Gymnostomum aeruginosum } \\
\text { Other species }\end{array}$ & + & . & 1 & . & . \\
\hline Syntrichia norvegica & + & . & 1 & 1 & . \\
\hline Mnium stellare & . & 1 & + & . & . \\
\hline Scapania cuspiduligera & . & . & . & . & 1 \\
\hline
\end{tabular}

Localities: All in Albania; 1-4: Maja e Jezercës; 5: Buni Jezercës. 


\section{BRYOPHYTE VEGETATION}

Two associations have been identified and referred to the bryosociological class Ctenidietea mollusci, that includes basophytic, mesophytic to meso-hygrophytic communities developed on rocks, rock crevices and rock outcrops covered by soil (PUGLISI \& PRIVITERA, 2012). A third community is referred to the bryo-chormophytic phytosociological class Montio fontanae-Cardaminetea amarae that includes the communities growing in shaded springs and montane streams.

\section{Pseudoleskeetum incurvatae Ježek \& Vondráček 1962 (Table 1)}

It is a meso-hygrophytic and meso-sciophytic, psychrophytic, exochomophytic community, generally settled in sheltered habitats on moist limestone rocks, crumbled boulders and gravelly soil having a thick layer of humus (PugLisi \& al., 2013a). In Albania this association was recognized in Maja e Jezercës and Buni Jezercës at elevations ranging between 1800 and $2500 \mathrm{~m}$,

Table 2

Solorino saccatae-Distichietum capillacei Reimers 1940

(Distichion capillacei, Ctenidietalia mollusci, Ctenidietea mollusci)

\begin{tabular}{lccc}
\hline Altitude $(\mathrm{m})$ & 2450 & 2450 & 2300 \\
Size of relevé $\left(\mathrm{cm}^{2}\right)$ & 40 & 50 & 50 \\
Cover $(\%)$ & 65 & 30 & 60 \\
Inclination $\left({ }^{\circ}\right)$ & 5 & 5 & 5 \\
Exposure & E & E & E \\
Relevé number & 1 & 2 & 3 \\
\hline
\end{tabular}

Characteristics

Distichium capillaceum

Tortella tortuosa

Encalypta streptocarpa

Distichium inclinatum

Mnium thomsonii

Other species

Syntrichia norvegica

Bryum schleicheri

Pseudoleskea incurvata

$\begin{array}{lll}2 & 2 & 3 \\ 3 & 1 & 1 \\ \cdot & 1 & \cdot \\ + & \cdot & 1 \\ + & \cdot & + \\ + & + & 1 \\ 1 & \cdot & + \\ \cdot & \cdot & 1\end{array}$

Localities: 1, 2: Maja e Jezercës (Albania); 3: Mt. Korab (Macedonia). in a vegetational context dominated by the $\mathrm{Cre}$ pidetalia dinaricae communities which are very rich in Circumboreal (Dryas octopetala, Draba aspera), Amphi-adriatic (Edraianthus graminifolius, Saxifraga marginata, Carex kitaibeliana, Artemisia eriantha, Trifolium noricum) and Balkan endemics (Valeriana bertiscea, Draba kummerlei, Arabis scopoliana, Saxifraga prenja, Danthoniastrum alpinum, Aubrieta croatica). The stand is floristically poor for the severe environmental conditions of the area. The characteristic species of the association is Pseudoleskea incurvata, a Boreal-montane species, which is accompanied by some species of higher units, such as Tortella tortuosa, Encalypta streptocarpa, Fissidens dubius and Gymnostomum aeruginosum. Synsystematically, the association is included in Ctenidion mollusci, order Ctenidietalia mollusci and Ctenidietea mollusci. It is a temperate-high montane-subalpine association, occurring on the mountains of Central-Western Europe (VADAM \& CAILLET, 2001) and also recently recorded in Greece (Puglisi \& al., 2013a).

\section{Table 3}

Cratoneuretum commutati Aichinger 1933

(Cratoneurion commutati, Montio-Cardaminetalia, Montio-Cardaminetea)

\begin{tabular}{|c|c|c|c|c|}
\hline Altitude (m) & 1850 & 1850 & 2600 & 2600 \\
\hline Size of relevé $\left(\mathrm{dm}^{2}\right)$ & 9 & 9 & 4 & 4 \\
\hline Cover $(\%)$ & 85 & 85 & 55 & 60 \\
\hline Inclination $\left({ }^{\circ}\right)$ & 70 & 70 & 60 & 80 \\
\hline Exposure & NW & NW & ENE & ENE \\
\hline Relevé number & 1 & 2 & 3 & 4 \\
\hline \multicolumn{5}{|c|}{ Characteristics of association } \\
\hline Palustriella commutata & 3 & 3 & 2 & 3 \\
\hline \multicolumn{5}{|c|}{ Characteristics of alliance, order and class } \\
\hline Cratoneuron filicinum & 2 & + & 1 & + \\
\hline Palustriella decipiens & 2 & 1 & . & . \\
\hline \multicolumn{5}{|c|}{ Ptychostomum pseudotriquetrum } \\
\hline var. pseudotriquetrum & 1 & 3 & 1 & 1 \\
\hline $\begin{array}{l}\text { Bryum schleicheri } \\
\text { Other species }\end{array}$ & . & . & 2 & 1 \\
\hline Philonotis caespitosa & . & . & 1 & + \\
\hline Polytrichum commune & . & . & . & 1 \\
\hline Pseudoleskeella nervosa & . & + & . & . \\
\hline
\end{tabular}

Localities: 1, 2: Buni Jezercës (Albania); 3, 4: Mt Korab (Macedonia). 


\section{Solorino saccatae-Distichietum capillacei Reimers 1940 (Table 2)}

In the rock fissures and cracks where soil accumulates, in shady and moist sites, both on the Maja e Jezercës (Albania) and Korab Mt (Macedonia), at elevations ranging between 2300 and $2400 \mathrm{~m}$, stands of the association Solorino saccatae-Distichietum capillacei were found. The floristic context of the sites of collection includes Ranunculus crenatus, Arabis flavescens, Achillea korabensis, Armeria rumelica and Saxifraga chrysosplenifolia. Ecologically, Solorino-Distichietum capillacei is a mesohygrophytic, sciophytic, basiphytic, and chasmophytic community (PugLisI \& al., 2013b). It is floristically characterized by the dominance of Distichium capillaceum, an arctic-montane species, and by the presence of some characteristics of higher ranks, such as Tortella tortuosa, Encalypta streptocarpa, Distichium inclinatum and Mnium thomsonii. The Solorino saccataeDistichietum capillacei is referred to the alliance Distichion capillacei, order Ctenidietalia mollusci and class Ctenidietea mollusci. It is a boreal-temperate-Mediterranean-alpinemontane association (Marstaller, 2006), occurring on the mountains of Central Europe while its previous records from the Mediterranean area are restricted to southern Italy, Sicily and Greece (Puglisi \& Privitera, 2012; Puglisi \& al., 2013a).

\section{Cratoneuretum commutati Aichinger 1933 (Table 3 )}

Stands of the association Cratoneuretum commutati were identified at Buni Jezercës and Korab Mt., where they grow on rocky outcrops permanently irrigated by base-rich, calcareous, cold waters. These stands belong to the community typical of mountain springs, actively forming travertine or tufa due to the deposition of carbonate on the moss carpet. It is a hygro-hydrophytic, basophytic association dominated by bryophytes. Physiognomically and floristically it is characterized by Palustriella commutata, guide species of the association, Ptychostomum pseudotriquetrum var. pseudotriquetrum, Cratoneuron filicinum, Palustriella decipiens and Bryum schleicheri, characteristic taxa of higher syntaxonomic units. This association is widespread in Europe, especially in the temperate zones. As far as the Mediterranean region is concerned it is known from mountain areas of Spain (GIL \& VARO, 1982; Fuertes LASALA \& MARTínEZConde, 1988; Heras \& al., 1989; GIL, 1997; GAVILÁN \& al., 2012), France (BARDAT \& HAUGUEL, 2002) and S Italy (BRULLO \& al., 2001).

\section{ACKNOWLEDGEMENTS}

We gratefully acknowledge the financial support provided by the Serbian Ministry of Science and Technological Development (project no. 173030, to Dmitar Lakušić). We warmly thank Z. Nikolov (Skopje, Macedonia) and Z. Bulić (Podgorica, Montegro) for their assistance during the field work. We also thank anonymous reviewers for the comments.

\section{SYNTAXONOMICAL SCHEME}

CTENIDIETEA MOLLUSCI v. Hübschmann ex Grgić 1980

Ctenidietalia mollusci Hadàc \& Šmarda ex Klika 1948

Ctenidion mollusci Ştefureac ex Klika 1948

Pseudoleskeetum incurvatae Ježek \& Vondráček 1962

Distichion capillacei Gjaerevoll 1956

Solorino saccatae-Distichietum capillacei Reimers 1940

Montio Fontanae-CARDAminetea AmaRAe Br.-Bl. et R. Tx. ex Klika et Hadač 1944 em. Zechmeister 1993

Montio fontanae-Cardaminetalia amarae Pawlowski 1928 em. Zechmeister 1993

Cratoneurion commutati W. Koch 1928

Cratoneuretum commutati Aichinger 1933 


\section{REFERENCES}

Bardat, J. \& Hauguel, J.C. -2002 - Synopsis bryosociologique pour la France - Cryptogamie Bryol. 23 (4): 279-343.

Barina, Z. \& Pifko, D. -2008 - Additions and amendments to the flora of Albania - Willdenowia 38: 455-464.

Beck, G. \& Szyszylowicz, I. - 1888 - Plantae à Dre Ign. Szyszylowicz in itinere per Cernagoram et in Albania adjacente anno 1886 lectae. Cracoviae, Universitatis Jagellonicae.

Bornmüller, J. - 1933 - Zur Flora von Montenegro, Albanien und Mazedonien - Mag. Bot. Lapok. 32: 109-142.

Braun-Blanquet, J.J. -1964- Pflanzensoziologie Grundzüge der Vegetationskunde, 3. Berlin, Springer.

Brullo, S., Spampinato, G. \& Scelsi, F. -2001- La vegetazione dell'Aspromonte. Studio fitodociologico - Reggio Calabria Laruffa Ed. 368 p.

Colacino, C. \& Sabovljević, M. - 2006- Bryophyte flora of Albania: a preliminary check-list - Cryptogamie Bryol 27 (4): 471-498.

Degen, A. \& Dröfler, J. - 1897 - Beitrang zur Flora Albaniens und Mazedoniens - Wien.

Düll, R. - 1983 - Distribution of the European and Macaronesian liverworts (Hepaticophytina) - Bryol. Beitr. 2: 1-114.

Düll, R. - 1984- Distribution of the European and Macaronesian mosses (Bryophytina) I - Bryol. Beitr. 4: 1-109.

Düll, R. - 1985 - Distribution of the European and Macaronesian mosses (Bryophytina) II - Bryol. Beitr. 5: 110-232.

Düll, R. - 1992- Distribution of the European and Macaronesian mosses (Bryophytina). Annotations and progress - Bryol. Beitr. 8/9: 1-223.

Düll, R., Ganeva, A., Martinčič, A. \& Pavletić Z. 1999 Contributions to the bryoflora of former Yugoslavia and Bulgaria - Bryol. Beitr. 11: 1-99.

Euro + Med Plant Base -2006 - The information resource for Euro-Mediterranean plant diversity - Retrieved September 3, 2012 from http://www.emplantbase.org/information.html

Filipovski G., Rizovski, R., Ristevski, P. - 1996- The characteristic of the climate-vegetation-soil zones (regions) in the Republic of Macedonia. Macedonian Academy of Sciences and Arts, Skopje.

Fuertes Lasala, E. \& Martinez Conde, E. - 1988 - Vegetación briofítica del macizo oriental de los Picos de Europa en Cantabria (España), I. Comunidades saxícolas, acuáticas y subacuáticas - Cryptogamie Bryol. L. 9: 109127.

Gavilán, R.G., Díez-Monsalve, E., Izquierdo, J.L., Gutiérrez-Girón, A., Fernández-González, F. \& SánchezMata, D. -2012 - An approach towards the knowledge of Iberian high-mountain calcareous grasslands - Lazaroa 33: 43-50.

Gil, J.A. - 1997 - Flora y vegetatión briofíticas de las Sierras de Cazorla y Segura (NE de Jaen, España) - Monogr. Fl. Veg. Béticas 10: 1-73.
Gil, J. \& Varo, J. - 1982 - Las alianzas Montion-Cratoneurion commutati en Sierra Nevada (España) - Doc. Phytosoc. N. S. 6: 369-386.

Grisebach, A. - 1843- Spicilegium Florae rumelicae et bithynicae. Vol. I - Braunsvigae. 407 pp.

Grisebach, A. - 1844- Spicilegium Florae rumelicae et bithynicae. Vol. II - Braunsvigae. 539 pp.

Hayek, A. - 1917- Beitrag zur Kenntnis der Flora des albanisch-montenegrinischen Grenzgebietes. Denkschrift der Akademie der Wissenschafte, Mathematisch Naturwissenschaft liche Klass, Wien 94: 127-210.

Heras-Ibáñez. J., Ros, R.M. \& Guerra, J. -1989-Flora y vegetación briofitica de la Sierra del Relumbrar (SW de Albacete, España) - Lazaroa 11: 149-175.

Herzog, T. -1919- Beiträge zur Bryogeographie Südosteuropas I. Mazedonien - Krypt. Forschung. Bayr. Bot. Ges. München Heft 4.

Hill, M.O., Preston, C.D., Bosanquet S.D.S. \& Roy, D. B. -2007- BRYOATT: Attributes of British and Irish Mosses, Liverworts and Hornworts - Cambridge, Centre for Ecology and Hydrology.

Košanin, N. - 1939- Über die Vegetation von Nordalbanien - Spomenik LXXXIX (Prvi Razed) 20: 75-105.

Lakušić, D, Stevanović, V, Bulić, Z, Jovanović, S, Tomović, G \& Vukojević, S. - 2004 - Florological and chorological contributions to the vascular flora of Montenegro Glas. Rep. Zav. Zašt. Prirode Muz. Podgorica 27-28: 33 42.

Marstaller, R. - 2006 - Syntaxonomischer Konspekt der Moosgesellschaften Europas und angrenzender Gebiete - Haussknechtia 13: 1-192.

Obuljen, A. - 1962 - The climate of Montenegro - Nolit. Pp. 24-46. Belgrade

Papp, B, Erzberger P., Marka, J. - 2010 - Contributions to the bryophyte flora of eastern Albania (Korça and Kolonja districts) - Studia Bot. Hung. 41: 61-88.

Privitera, M. \& Puglisi, M. - 1997 - Noteworty orophilous mosses from Mount Etna (Sicily) - Bocconea 5 (2): 905-911.

Privitera, M. \& Puglisi, M. - 2002- Some interesting records for the Italian moss flora - Cryptogamie Bryol. 23 (2): 171-179.

Privitera, M. \& Puglisi, M.-2009- The circum-Sicilian islands as important refuge areas for some remarkable bryophytes - Pl. Biosyst. 143 (S1): 126-135.

Puglisi, M. -2008 - La Fitosociologia delle Briofite in Italia - In: AA.VV. Biologia ed Ecologia delle Briofite. Pp. 257-267. Antonio Delfino E., Roma.

Puglisi, M. - 2009- New interesting records to the moss flora of Sicily (Italy) - Cryptogamie Bryol. 30 (3): 395 398.

Puglisi, M. - 2010 - Contribution to the bryophyte vegetation of the Pelagian Archipelago: the Island of Linosa (Sicily Channel, Italy) - Nova Hedw. Beih. 138: 257282. 
Puglisi, M. \& Privitera, M. - 2012- A synopsis of the Italian bryophyte vegetation - Cryptogamie Bryol.33(4): 357-382.

Puglisi, M., Privitera, M. \& Di Pietro, R. -2009- New interesting bryophyte records from Pollino National Park (Southern Italy) - Fl. Medit. 19: 5-8

Puglisi, M., Privitera, M. \& Di Pietro, R. - 2011 - New records of arctic montane and alpine mosses from Central Italy - Cryptogamie Bryol. 32(1): 75-82.

Puglisi, M., Kürschner, H. \& Privitera, M. -2013a- Saxicolous bryophyte communities of mountain areas of Greece: phytosociology, ecology, life forms and life strategies - Nova Hedwigia 97 (1-2): 159-178.

Puglisi, M., Kürschner, H. \& Privitera, M. -2013bSyntaxonomy, life forms and life strategies of the bryophyte vegetation of the Carnic Alps (NE Italy) Nova Hedwigia 96 (3-4): 325-349.

Puglisi, M., Tamburino, A., Privitera, M. -2012- Additions to the moss flora of Greece - Cryptogamie Bryol. 33 (4): 383-389.

Rakaj, M. - 2009- Floristic and chorological news from north Albania - Bot. Serb. 33 (2): 177-183.

Ros, R. M., Mazimpaka, V., Abou-Salama, U., Aleffi, M., Blockeel, T. L., Brugués, M., Cano, M. J., Cros, R. M., Dia, M. G., Dirkse, G. M., El Saadawi, W., Erda ̆, A., Ganeva, A., Gonzalez-Mancebo, J. M., Herrnstadt, I., Khalil, K., Kürschner, H., Lanfranco, E., Losada-Lima, A., Refai, M. S., Rodriguez-Nuñez, S., Sabovijević, M., Sérgio, C., Shabbara, H., Sim-Sim, M. \& Söderström, L. - 2007- Hepatics and Anthocerotes of the Mediterranean, an annotated checklist - Cryptogamie Bryol. 28 (4): 351-437.

Ros, R. M., Mazimpaka, V., Abou-Salama, U., Aleffi, M., Blockeel, T. L., Brugués, M., Cros, R. M., Dia, M. Giovanna, Dirkse, G. M., Draper, I., El-Saadawi, W., Erdağ, A., Ganeva, A., Gabriel, R., González-Mancebo, J. M., Granger, C., Herrnstadt, I., Hugonnot, V., Khalil, K., Kürschner, H., Losada-Lima, A., Luís, L., Mifsud, S., Privitera, M., Puglisi, M., Sabovljević, M., Sérgio, C., Shabbara, H. M., Sim-Sim, M., Sotiaux, A., Tacchi, R.,
Vanderpoorten, A. \& Werner, O - 2013- Mosses of the Mediterranean, an annotated checklist - Cryptogamie Bryol. 34 (2): 99-283.

Sabovljević, M., Ganeva, A., Tsakiri, E. \& Ştefănuţ, S. 2001 - Bryology and bryophyte protection in the southeastern Europe - Biol. Conserv. 101: 73-84.

Sabovljević, M., Natcheva, R., Dihoru, G., Tsakiri, E., Dragićević, S., Erdağ, A. \& Papp, B. -2008- Check-list of the mosses of SE Europe - Phytol. Balcan. 14(2): 207-244.

Söderström, L., Urmi, E. \& Váña, J. -2002 - Distribution of Hepaticae and Anthocerotae in Europe and Macaronesia - Lindbergia 27: 3-47.

Stevanović, V., Tan, K. \& Iatrou, G. - 2003- Distribution of the endemic Balkan flora on serpenitine. Obligate serpentines endemics - Plant Syst. Evol. 242: 149170.

Stevanović, V, Vukojičić, S, Šinžar-Sekulić, J, Lazarević, M, Tomović, G \& Tan, K. -2009- Distribution and diversity of Arctic-Alpine species in the Balkans - Plant Syst. Evol. 283: 219-235.

Tsakiri, E., Karagiannakidou, V. \& Babalonas, D. -1998General Overview and Chorological Analysis of Bryophytes in Northern Greece (Macedonia and Thrace) - In: Tsekos, I. \& Moustakas, M. (Eds). Progress in Botanical Research, Proceedings of the 1st Balkan Botanical Congress. Pp. 165-168. Kluwer Academic Publishers.

Vangjeli, J., Ruci, B., Mullaj, A., Paparisto, K. \& Qosja, X.H. -2000 - Flora e Shqiperise, Vol. 4 (in Albanian) Akademia e Shkencave e Republikes se Shqiperise, Instituti i Kerkimeve Biologjike, Tirane. 502 pp.

Vadam, J.-C. \& Caillet, M. - 2001 - Le Ptichodio plicatiLescureetum incurvatae, une nouvelle association bryophytique des massifs montagneux calcaires du Jura et des Alpes - Cryptogamie Bryol. 22: 187-198.

Vranaj,A. - 1990 - Gjeologiia e Shqiperise (in Albanian) Tirana. $420 \mathrm{pp}$.

Weber, H.E., Moravec, J. \& Theurillat, J.P. - 2000 - International Code of Phytosociological Nomenclature. 3rd edition - J. Veg. Sci. 11: 739-768. 